\title{
Conf-9107138--4
}

UCRL-JC- -108002

DE92 002194

\section{Shock-Interface Interaction: Current Research on the Richtmyer-Meshkov Problem}

\section{Rupert}

This paper was prepared for presentation at

July 21-26, 1991

Juily 17, 1991

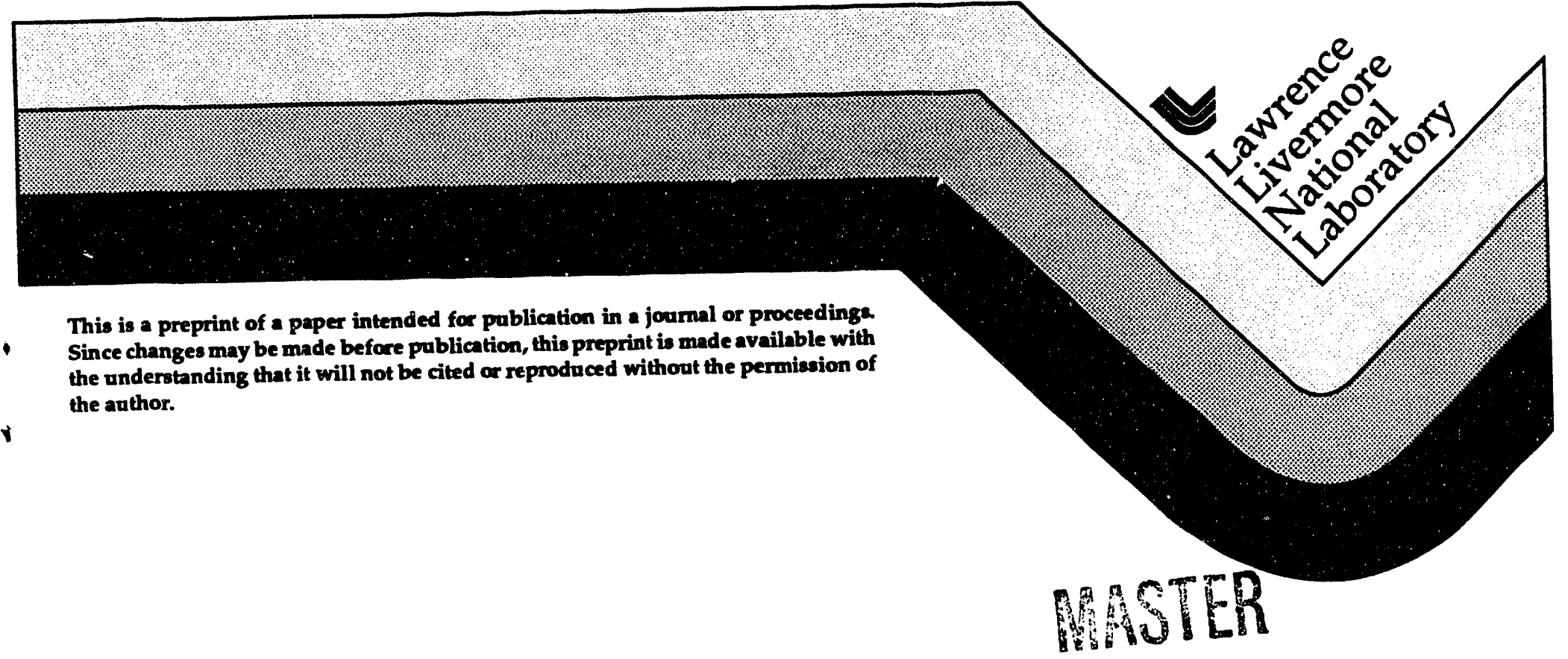

This is a preprint of a paper intended for publication in a jourmal or proceedinga Since changes may be made before publication, this preprint is made available with the understanding that it will not be cited or reproduced without the permisaion of the author.

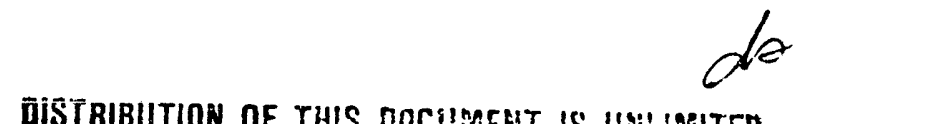


DISCI.AIMER

This document was prepared as an account of work sponsored by an agency of the United States Government. Neither the United States Government nor the University of California nor any of their employees, makes any warranty. express or implied, or assumes any legal liability or responsibility for the accuracy, completeness, or usefulness of any information, apparatus, product, or process disclosed, or represents that its use would not infringe privately owned rights. Reference herein to any specific commercial products, process, or service by trade name, trademark, manufacturer, or otherwise, does not necessarily constitute or imply its endorsement, recommendation. or favoring by the United States Government or the University of California. The views and opinions of authors expressed herein do not necessarily state or reflect those of the United States Government or the University of California, and shall not be used for advertising or product endorsement purposes. 


\title{
Shock-Interface Interaction: \\ Current Research on the Richtmyer-Meshkov Problem
}

\author{
V. Rupert
}

The problem of shock refraction at a material interface has been studied both experimentally and numerically. Recent work (Henderson 1989) has provided insight into the complex problem of anomalous refraction. Emphasis has been placed on the pattern of rifiected and refracted waves, including precursors. However, the evolution of the interface itself is of considerable interest in problems ranging from inertial confinement fusion to astrophysics (Klein 1989) and has generated a flurry of activity that encompasses the subject of compressible turbulence.

The basis for the study of the evolution of a shocked interface stems from the question of the Rayleigh-Taylor (RT) instability (Taylor 1950). Starting in the late 18th century, the stability of an interface submitted to gravitational forces was investigated for the case in which the density of one of the materials across the interface was negligible compared to the other. Taylor analyzed the case in which the Atwood number (ratio of the difference of the densities to their sum) is less than 1 , and the acceleration of the system is constant. He determined that the interface was unstable to small perturbations only if the direction of the acceleration normal to the interface coincides with that of the density gradient. Richtmyer (1960) extended Taylor's analysis to the case of an impulsive acceleration. His results implied that the interface would be unstable irrespective of the relative orientation of the velocity impulse and the density gradient. His predictions were verified experimentally by Meshkov (1969), and the Richtmyer-Meshkov (RM) instability became a subject of research in its own right. Experimental, numerical, and theoretical works address this problem.

To simnlify the following discussion, the case in which the shock is incident from the low-density sic ' $^{\circ}$ on a interface with a denser material will be referred to as RT unstable. An RT stable case corresponds to a shock incident from the high-density side on an interface with a lighter material.

Richtmyer's original prediction was that, for a discontinuous interface, the perturbation would grow linearly with time as

$$
a=a_{0}(1+A k V t),
$$

where $a_{0}=$ the initial amplitude, after shock compression,

$A=$ the Atwood number, after shock compression,

$V=$ interface velocity, and

$k=2 \pi / \lambda=$ wave number.

\section{Experimental work}

The RM problem has been studied with both shock-tube and laser experiments. In this paper, only shock-tube work is considered.

\subsection{Discontinuous interface with single-wavelength perturbations}

Meshkov's pioneering experiments were designed to test Richtmyer's predictions, which strictly applied only to a single wavelength perturbation of an interface. The assumptions were that (1) $a_{0} / \lambda$, the initial amplitude-to-wavelength ratio of the 
perturbation, was small ( $<<1)$ so that the equations could be linearized, (2) the incident shock was normal to the average (unperturbed) interface position, and (3) the flow was nearly incompressible arter shock passage. The experiments confirmed that (1) the rate of change of the perturbation's amplitude was constant, as opposed to the exponential growth of the RT instability, and (2) for an RT stable configuration, the perturbation changed phase. However, the measured growth rates were as much as a factor of 3 less than predicted by Richtmyer. Benjamin (1988) performed similar experiments and confirmed Meshkov's results. Actually, to facilitate observations, the experiments were conducted with $0.05 \leq a / \lambda \leq 0.1$ (before shock compression), so that the perturbations became nonlinear within the observation time. In addition, the gases used in the experiments were separated by a thin membrane. Some of the energy transferred from the shock to the perturbed interface could have been lost to the membrane, which was shattered and accelerated with the flow. Finally, particularly when helium was used, diffusion across the membrane could have reduced the Atwood number. Any of these experimental factors could account for the lower growth rate.

The persistence of constant growth beyond the linear phase is reminiscent of results obtained by Youngs (1984) and Read (1984) for the RT instability. They found that, at late times, the amplitude of the perturbation was proportional to the average displacement of the interface. This result can be obtained directly from dimensional analysis, if one assumes that memory of the initial perturbation is lost. In the case of a shock, the interface velocity is constant, and so is the perturbation growth rate. However, the proportionality factor cannot be expected to be identical to that of the small-amplitude indicate that, to first order, between small-amplitude growth and the asymptotic growth rate.

Meshkov's and Benjamin's experiments were conducted for weak shock waves (incident Mach number $\approx 1.3$ ). Aleshin (1988) and Zaitsev (1991) studied the case of a stronger shock (incident Mach number 3.5) and found better agreement with Richtmyer's prediction. In this case, the compressed value of $a / \lambda$ was smaller so that a true linear phase was observed. In addition, the thicker membrane used provided a better definition of the Atwood number. However, if the membrane itself had been the origin of the discrepancy observed in Meshkov's and Benjamin's experiments, a similar discrepancy should have been observed in Aleshin's experiments. Indeed, work of Smith (1989) indicates that thick membranes can have a significant effect on the flow. A closer look at the data from these various experiments should shed some light on the role of membranes, which tend to be blamed for much of the disagreement between experiments and numerical results.

Other high-Mach-number experiments with single wavelengths were reported by Vasilenko (1988), who used an electromagnetic shock tube and obtained better agreement with theoretical estimates. In this case, however, a strong deceleration of the flow behind the shock resulted in a significant RT phase, so the results are not a direct test of the RM problem.

\subsection{Discontinuous interface with random-wavelength perturbations}

While the study of single-wavelength perturbations provides some insight into the RM phenomenon, usual interfaces are nominally flat but comprise a spectrum of smallamplitude perturbations. The spectrum is often near white, in which case the component of the perturbation with the smaller wavelength grows the fastest, according to Richtmyer's formula. As the value of $a_{0} k$ reaches $\sim 0.4$, its growth rate starts to decrease, 
and the next component takes over, so that a nonlinear phase is attained quite rapidly. In the process, mode coupling occurs, and the spectral content of the interface is modified. When several dominant wavelengths are present, larger scales develop by means of a bubble-competition mechanism (Zufiria 1988) and vortex pairing. Simultaneously, a breakup of the perturbation occurs, generating much smaller scales, and the flow becomes fully turbulent. In such cases, it is not possible to distinguish the shape of the perturbed interface; rather, a mixing region is obtained, where quantities such as the overall width or average concentration profile can be determined. Figure 1 (Rupert,1991) illustrates a similar evolution for a small number of wavelengths.

Experiments were performed with a nominally flat membrane separating the test gases in a shock tube (Andronov 1976; Zaitsev 1985; Brouillette 1988; Houas 1988; and Cavailler 1991). One of the most noticeable result from these experiments is the small growth rate following the passage of the incident shock through the interface and its significant increase after the incident shock reflects off the end wall. In the latter case, the reflected shock interacts with a pre-existing turbulent region that results from the incident shock, transfers energy to it, and increases the turbulence, thus accounting for the increased growth rate of the mixing layer. Experimental results for similar initial conditions are the center of an unresolved controversy resulting from the difference in growth rates quoted by Andronov (1976) and Brouillette (1988). Andronov's values are about an order of magnitude larger than Brouillette's, whose data were recovered by Cavailler (1991). Two possible causes for the discrepancy have been proposed: the influence of the boundary layer (Brouillette) and the effect of the membrane (Meshkov). Because of shock/boundary-layer interaction, large vortices develop along the walls after reshock and grow at a much faster rate than do the perturbations at the center of the shock tube. Because schlieren is an image of the density gradients integrated over the width of the tube, separation of the signature of the main flow from that at the boundary is sometimes difficult. Brouillette's measurments of wal! vortices gave growth rates similar to those quoted by Andronov.

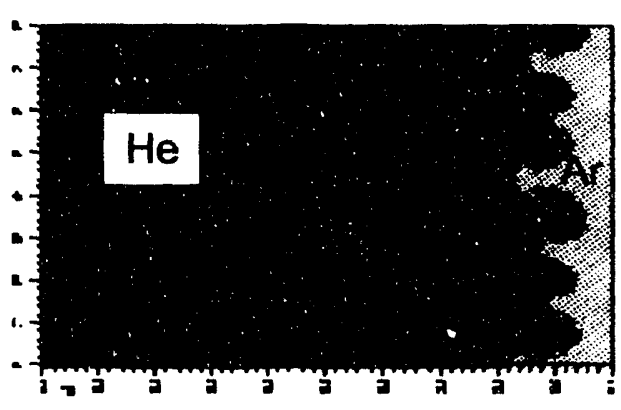

(a) Log density, $0.05 \mathrm{~ms}$

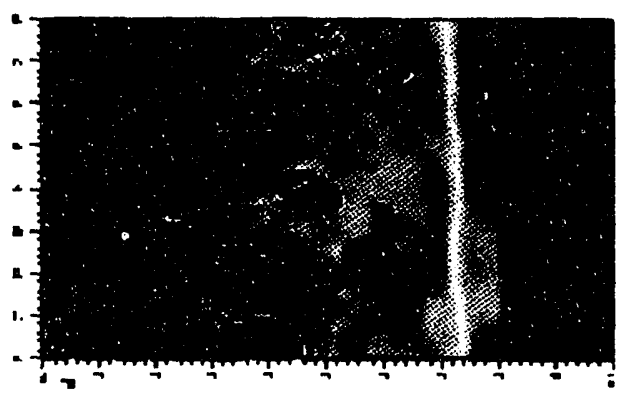

(b) Density, $0.35 \mathrm{~ms}$
Figure 1. The RM unstable interface traverses several stages, following an initial linear growth. These simulations were performed for a Mach 3.45 shock in Ar incident on an Ar/He interface. A multiwavelength perturbation was imposed on the interface. (a) The incident shock has crossed the interface; vortices are developing at the tip of the Ar spikes. (b) After multiple shock transit, larger scales resulting from vortex pairing as well as small-scale turbulence have developed. 
Moreover, transverse waves from wall vortices interact with the center flow and influence its evolution. By such a mechanism, differences in wall smoothness could result in different growth rates of the perturbed interface.

\subsection{Diffuse interfaces}

In a shock-tube environment, the materials impinging on the interface are gases, and it is difficult to obtain a discontinuous interface without some solid membrane whose effect is as yet unresolved. However, because a characteristic of RT or RM instability is the Atwood number, a diffuse interface should result in lower growth rates. This was indeed predicted by Lelevier (1955) for the RT case and reviewed by Mikaelian (1985) for the RM case, in which an incompressible flow behind the shock was assumed. Two facilities have been built to test these predictions experimentally. Both are vertical shock tubes in which the test gases initially are separated by a retractable plate. Just prior to shock passage, the plate is withdrawn, and the gases interdiffuse. In the Caltech tube (Brouillette 1988), the shock is incident from the top, so that RT unstable cases are instigated; in the Vaujours tube (Cavailler 1989), the shock is incident from the bottom. In accordance with predictions, the experimental growth rate after passage of the incident shock is indeed quite small (too small, in fact, to be measured reliably), but again the growth rate increases markedly after passage of the reflected shock. These observations are true for either nominally planar or quasi-single-wavelength interface shapes. For the latter, Brouillette proposed a modification of Richtmyer's formula, in which a growthreduction factor depends on the Atwood number and the initial thickness of the diffusion zone. His calculations imply growth rates as small as $1 / 20$ th of the growth for a discontinuous interface between the same gases. Fair agreement was obtained between his experiments and the formula he proposed, even when the perturbation became slightly nonlinear.

\subsection{Discontinuous interface with large-scale perturbations}

While the preceding experiments examined the evolution of an interface with small initial perturbations, a few experiments have investigated the large-pertirbation case. Two types of experiments were conducted along these lines.

Large-scale sawtooth perturbations can be simulated by studying the interaction of a planar shock with a tilted interface. The same information on the initial interiace deformation was obtained in studies of irregular shock refraction through tilted interiaces (Puckett 1989). Haas and Sturtevant (1985) examined the late-stage development of such an interface, including the case of two equally tilted interfaces containing different layers of gas. The large-scale perturbation defined by the tilt evolved as expected (with an inversion of the tilt angle for the RT stable case), and large-scale vortices developed at the tips. Along the interface, in the nominally planar region, small-scale perturbations evolved as a result of both RM and Kelvin Helmholtz instabilities, and a wide mixed region appeared. Yang and Zabuski (1989) performed numerical simulation of these experiments and emphasized the role of dipolar vortex binding in breaking through a thin layer.

Large-scale perturbations of a different kind were also investigated, in spheres or cylinders traversed by a planar shock. Haas (1983) studied this configuration in the context of shock refraction, using schlieren imaging and soap bubbles or thin plastic membranes to separate the gases. Recently, more detailed data on cylindrical perturbations were obtained with buoyant cylindrical jets by Jacobs (1991), who used laser induced fluorescence of a biacetyl dopant, and by Budzinski (1991), who used Rayleigh scattering. These latter experiments bypassed the difficulty of accounting for the 
effect of the membrane and, with the use of a laser sheet, provided data on a thin slice of the cylinder, yielding more precise information on the two-dimensional evolution of the interface. These experiments show simultaneously the evolution of RT-stable and -unstable interfaces, subjected to varying vorticity, and clearly show the deformation of the cylinders and formation of ring vortices.

\subsection{Diagnostics}

An important aspect of the work on RM instability is the development of a predictive capability, so that its influence on the structure of the interstellar medium or the efficiency of an inertial confinement fusion implosion can be assessed. To do so requires experimental data that can be compared directly with theories or numerical computations. In most experiments described thus far, schlieren imaging was used to provide the timedependent width of mix layers. Observation through boundary layers could influence the data, and concentration gradients of the individual components could not be obtained. Other diagnostic techniques are being developed to provide complementary or new information. These include laser scattering techniques (Meshkov 1989; Benjamin 1989; Jacobs and Budzinski; 1991), infrared absorption and emission (Houas 1988), holography (Stearman 1989), and x-ray absorption (Bonazza and Sturtevant 1991).

\section{Numerical simulations}

Numerical simulations of the RM problem have been performed with a variety of techniques: two-dimensional calculations using purely Eulerian meshes (Besnard 1989; Wehner 1989), Eulerian meshes with interface tracking, Eulerian meshes with adaptive mesh refinement (Rupert 1989, 1991), arbitrary Lagrangian Eulerian meshes (Rauenzahn 1989; Griswold 1991), semi-Lagrangian meshes with interface tracking (Cowperthwaite 1989), and front-tracking methods (Glimm 1991); three-dimensional calculations using Eulerian codes (Cowperthwaite 1989; Wehner 1991).

All the calculations assume inviscid fluids. Mesh size limitations restrict the type of perturbations that can simulated. In general, a single wavelength or only a small number of wavelengths are used, with $a_{0} / \lambda$ ranging from 0.3 to $7 \%$. The meshes are defined in such a way as to ensure a good resolution of all the initial wavelengths. However, as the perturbation crosses through a nonlinear stage into the fully turbulent regime, smaller scales develop and eventually cannot be resolved. Adaptive-mesh and interface-tracking schemes are least susceptible to this loss of resolution but are limited nonetheless by the growing size of the problems versus the finite size of computer memories.

A few calculations have been initiated with small random perturbations (Besnard 1989) but present problems of convergence.

\subsection{Simulations of single- and multi-wavelength perturbations}

The single-wavelength calculations are in good agreement. A set of problems was run for air $\rightarrow \mathrm{He}$ at $\mathrm{M}=1.24$, air $\rightarrow \mathrm{SF}_{6}$ at $\mathrm{M}=1.32, \mathrm{Ar} \rightarrow \mathrm{He}$ at $\mathrm{M}=3.45$ and $\mathrm{He} \rightarrow \mathrm{Ar}$ at $M=2.77$, where $M$ is the Mach number of the incident shock. The $M=1.24$ case was a simulation of one of Benjamin's expcriments. The simulations showed a quasilinear growth after passage of the incident shock, as well as after the first reflected shock (Fig. 2, Rupert, 1989). The calculated growth rate just after passage of the incident shock through the interface ranges from 75 to $80 \mathrm{~m} / \mathrm{s}$ and was obtained by determining the growth of the layer containing a 5:95 ratio, per volume, of the constituents. (Note that this definition corresponds to $\sim 1.98$ times the amplitude for a sinusoidal perturbation.) This 


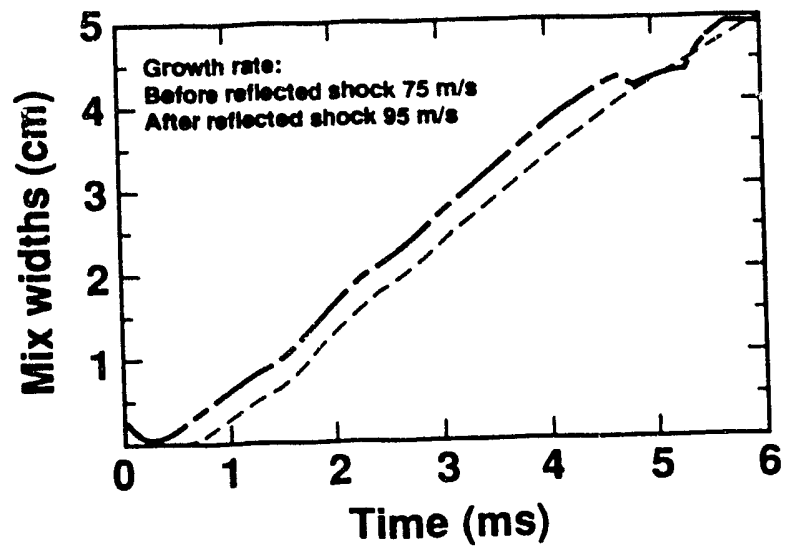

Figure 2. Numerical simulation of single (- $)$ - and multiple (- - - )wavelength perturbation growth at an air/He interface subjected to a Mach 1.24 shock incident from the air side. The growth rate is almost constant after both incident shock and reshock.

rate compares well with a prediction of $83 \mathrm{~m} / \mathrm{s}$ based on Richtmyer's formula, but it is much larger than the experimental value of $38 \mathrm{~m} / \mathrm{s}$. Because the initial conditions of the calculations and the experiments were identical, the discrepancy cannot result from the fact that the perturbation rapidly reached a nonlinear phase.

The influence of the membrane is difficult to simulate; because its density greatly exceeds that of the gases, its inclusion in the calculations necessitates both an extremely fine mesh and a simulation of the strength of the membrane material. In attempting to include it in the calculations, researchers usually resort to simulating it with a dense "gas" layer. Benjamin (1991) reports the results of such a calculation and concludes that the membrane should have a negligible effect.

The remaining obvious possible source of differences between calculations and experiments is the boundary layer, which is ignored in most calculations, where the shock tube's walls are treated as reflecting boundaries. This aspect of the problem was studied by Cloutman (1990), who used both no-slip and slip conditions at the walls. His results indicate little difference in the growth rates in the bulk of the flow, although additional vorticity was generated at the walls in the no-slip case. Thus the puzzle remains unsolved. Better ways of treating membranes and boundary layers need to be implemented, and simulations of the experiments without a membrane also need to be performed.

Results from simulations of the Benjamin problem, using an AMR code, are shown in Fig. 3 (Rupert,1989) for a multi-wavelength initial perturbation. Here, the evolution of the vorticity is shown at various times after arrival of the reflected wave at the interface. For the earliest time, the divergence of the velocity is also shown and displays, to the right of the interface, the location of the primary shock after it has been transmitted through the interface, reflected off the end wall, and retransmitted through the interface. Relative to the interface perturbation, the shock is almost flat. The secondary shock reflected off the interface is seen on the left of the interface and is also rather flat. Throughout the flow field can be seen a complex pattern of expansion and compression or shock waves resulting from the reflection and refraction at the interface of the incident shock and shocks reflected from the end wall. The near planarity of the strongest shocks results from the coalescence of the curvilinear shocks transmitted through the wavy interface. The vorticity, however, is seen to be concentrated at the interface, primarily at the tips of the spikes of denser fluid. As the perturbation grows, vortex pairing occurs, creating larger-scale structures. The successive shocks reflected off the end wall cross the interface area and increase the vorticity. 


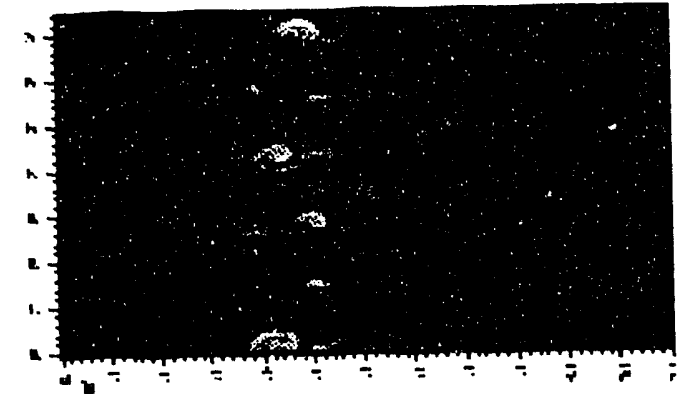

(a) Vorticity, $0.22 \mathrm{~ms}$

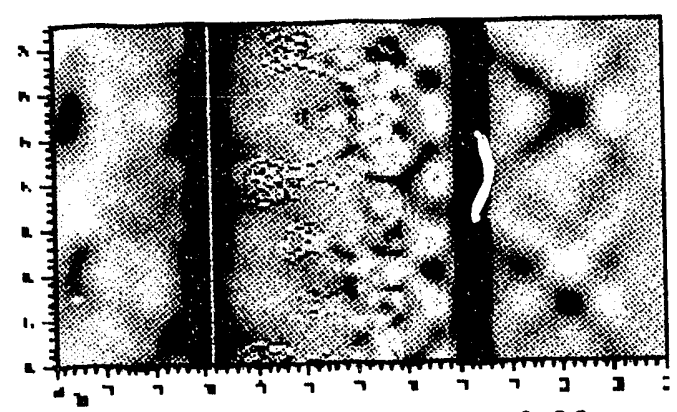

(b) Divergence (Velocity), $0.22 \mathrm{~ms}$

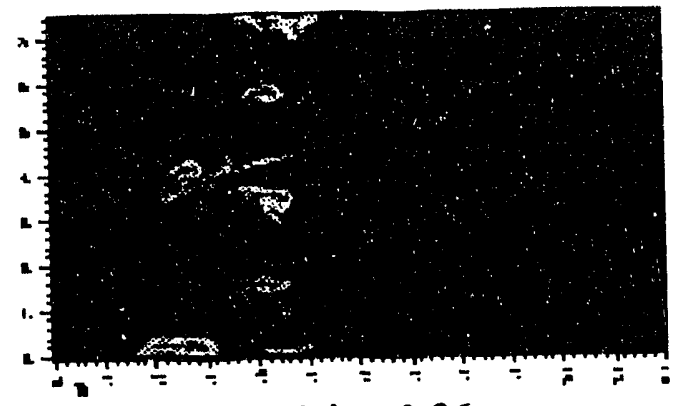

(c) Vorticity, $0.36 \mathrm{~ms}$

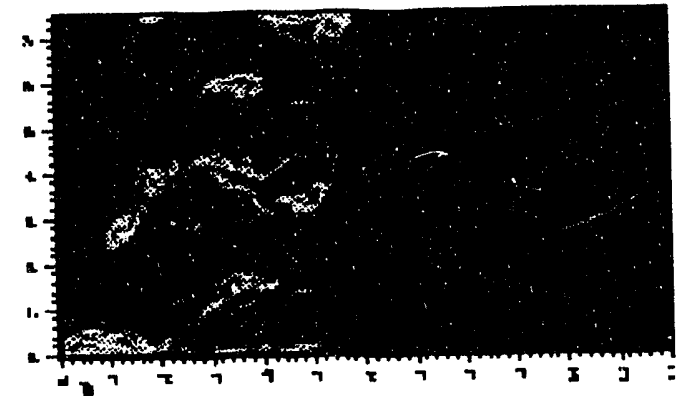

(d) Vorticity, $0.51 \mathrm{~ms}$

Figure 3. Numerical simulation of the evolution of a multiple-wavelength perturbation growth at an air/He interface subjected to a Mach 1.24 shock incident from the air side. (a), (c), and (d): Vorticity maps at various times, showing vorticity concentrated near the tips of the air spikes and vortex pairing at late time. (b): Map of the divergence of the velocity at the same time as in (a). It shows an almost planar transmitted and reflected shock, in spite of the proximity of a highly distorted interface.

From such calculations, the Reynolds stresses can be computed as a function of the position along the shock tube axis, and the turbulent energy (as the integral of the Reynolds stress tensor trace) is determined as a function of time. As Fig. 3 implies, the turbulent energy is concentrated in the mixed layer. As the first shock crosses the interface in these calculations, which neglect viscosity, turbulent energy is generated, diffuses, and decays slowly. At the arrival of the second shock, the turbulent energy increases by more than a factor of 10 as energy is transferred from the shock to a pre-existing turbulent region (Fig. 4, Rupert, 1991). In essence, the energy transfer is now more a result of small, random density fluctuations than of large density differences caused by different gases. Similar results were extracted from all numerical simulations.

An imprortant result of the 2-D simulations is the late-time RM growth rate's sensitivity to the initial conditions. This result marks a break from RT instability, where asymptotic growth rate has been shown bnth experimentally (Read 1984) and numerically (Youngs 1984) to be independent of the initial perturbation. Caution must be exercised, however, in extrapolating these findings to all situations because, in most experiments and simulations, the distance between the interface and the end wall was so short that the mixed layer was not fully turbulent before arrival of the reflected shock. 


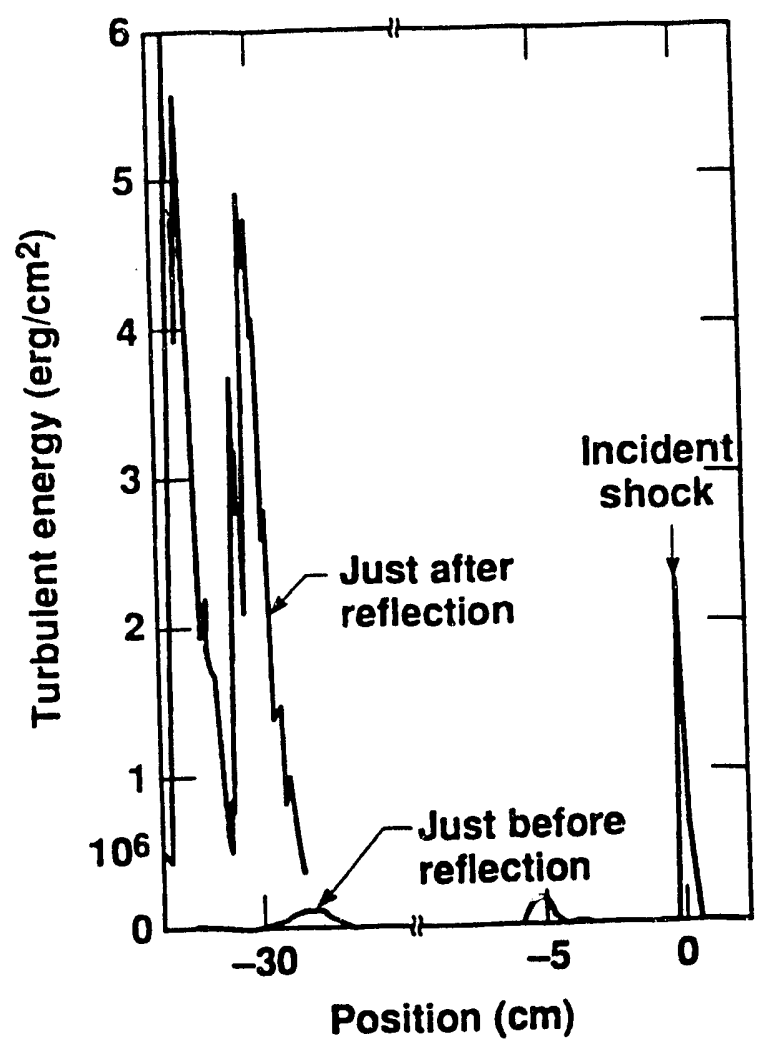

Figure 4. Snapshots of the turbulent energy at various times, resulting from the interaction of a Mach 2.7 shock in He incident on an Ar/He interface. Numerical simulations were performed with a single-wavelength perturbation. Following shock passage and turbulence generation, the turbulent energy diffuses across the inter-face, and the total energy decreases slightly. The reflected shock results in a $20 \times$ increase in total turbulent energy.

\subsection{Simulation of a perturbed gas layer}

Yang and Zabuski (1989) simulated gas layers for tilted planar interfaces. Here, simulations of a gas layer with interfaces normal to the shock-tube axis and comprising multiple-wavelength small-scale initial perturbation are presented (Rupert, 1989). This simulation was performed with an Adaptive Mesh Refinement code developped by Colella (Puckett, 1989). The configuration studied was proposed by Youngs. A weak shock, at $M=1.23$, is incident from air into an R22 (refrigerant) layer. The first interface, $I_{1}$, traversed by the shock is therefore RT unstable, while the second, $I_{2}$, is RT stable. Figure 5(a) shows the initial interface shape, with identical perturbations. In Fig. 5(b), the shock is halfway within the layer. The perturbation has grown on $I_{1}$, but it is still nearly linear. In Fig. 5(c), $I_{2}$ has reversed phase and has reached a highly nonlinear configuration, while $I_{1}$ is starting to show vortex pairing or mode coupling. At this point, the shock reflected off the end wall has just crossed the layer so that $I_{1}$ is RT stable and is changing phase, the mushroom heads are flattening, and $I_{2}$ is RT unstable and is continuing to grow. Little cross coupling is apparent between the interfaces at this point. In Fig. 5(d), the coupling between the interfaces is visible, although the layer has not yet broken through. The attempt of the highly complex shape of $I_{1}$ to change phase has led to a turbulent configuration.

Although this simulation involves a layer and (at late stages) cross coupling between the interfaces tends to influence the evolution of the interfaces, these results illustrate (in greater detail than the preceding simulations) the nonlinear phase of perturbation growth. Indeed, here, the end wall is sufficiently far from the interfaces to allow them to evolve to a highly nonlinear phase before the reflected shock arrives. Simulations of this kind, with a single interface, still need to be performed in order to study the question of sensitivity to initial conditions. 


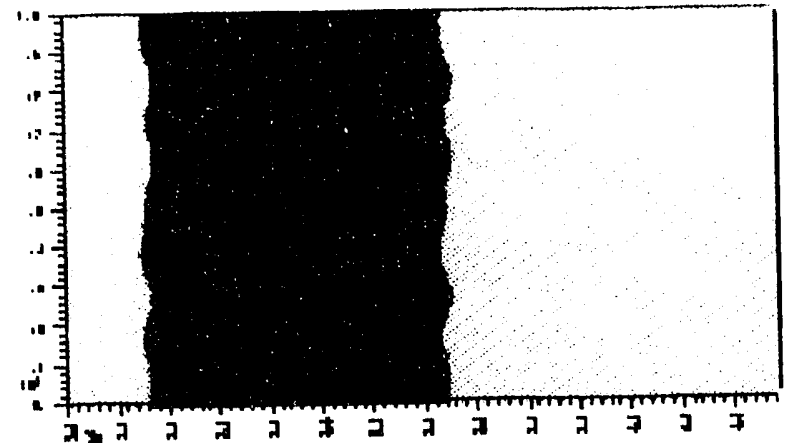

(a) Volume fraction, $0 \mathrm{~ms}$

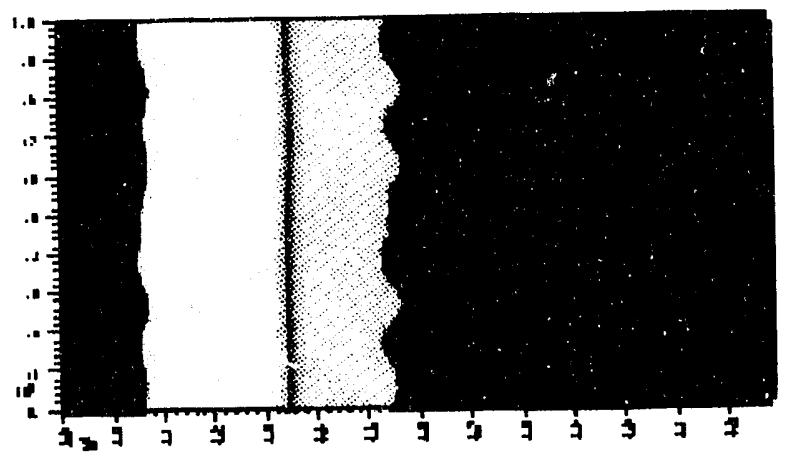

(b) Log density, $0.22 \mathrm{~ms}$

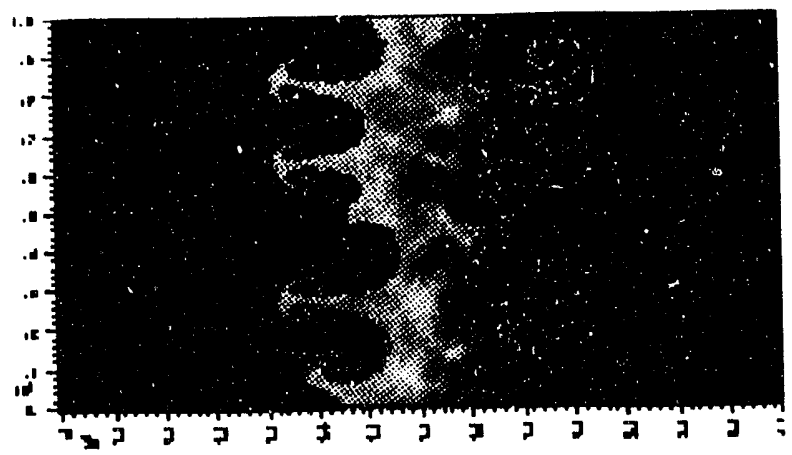

(c) Volume fraction, $1.91 \mathrm{~ms}$

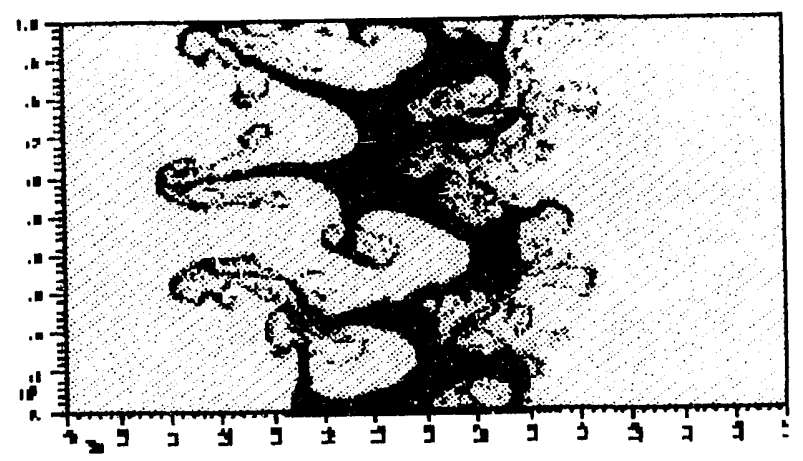

(d) Volume fraction, $2.55 \mathrm{~ms}$

Figure 5. Numerical simulation of an R22 layer in air, with multiple-wavelength perturbations of the layer boundaries. Incident shock Mach number was 1.23. (a) The initial volume fraction of air. (c) and (d) The volume fraction after the incident and reflected shocks have crossed the layer. (b) The density distribution when the incident shock is still within the layer.

\subsection{Three-dimensional simulations}

Two-dimensional calculations have been quite helpful in understanding the evolution of shocked interfaces. However, because part of the mixed layer develops turbulent characteristics, it is probable that the 2-D growth rates do not exactly reflect the real 3-D growth observed in the experiments. Several researchers (Cowperthwaite 1989; Sahoda 1990; Wehner 1991) have attempted to follow the evolution of the perturbations in 3-D.

Even to a larger extent than 2-D simulations, 3-D simulations are limited by the number of mesh zones that can be used, but progress has been made, particularly in the computation of single-wavelength perturbations. A simulation of the experimental configuration in which a cylindrically corrugated membrane separates the gases would require a 3-D calculation wherein a single wavelength is imposed in one direction and small random perturbations are superimposed in the perpendicular direction. Because of the small number of zones available, such random perturbations cannot be well resolved. Thus, 3-D simulations tend to be conducted with either a cylindrically symmetric, singlewavelength per urbation (Sahoda and Wehner) or the product of single wavelengths in two orthogonal directions (Cowperthwaite and Wehner). Calculations for a random perturbation have been reported by Youngs (1990) for the milder case of RT instability. 
Cowperthwaite's results indicate a higher growth in 3-D than in 2-D after multiple reshocks but appear to give the same growth rate after the incident shock and the first reflected shock. These results are in conflict with Wehner's, who in first analysis, finds slightly lower growth rates. Differences in the type of initial perturbations may account for the different results.

\section{Modeling}

The most important result of studies of RM instability is to provide a data base against which simple models can be calibrated and verified. Indeed, full 3-D simulations of astrophysical problems, or miniature ICF targets, are quite expensive in money, time, and computer memory. While a few cases can be run, it would be prohibitive to investigate a large number of scenarios to reach an acceptable configuration. Hence, much emphasis has been placed on developing models that can describe the evolution of shocked interfaces in terms of basic hydrodynamic equations, supplemented by 1-D evolution equations of a small number of additional variables.

The linear phase of perturbation growth can be described by Richtmyer's equations for the width of the mixed region. His formalism has been extended to describe the nonlinear evolution of a random perturbation. Several such extensions have been proposed (Crowley 1983; Neuvazhaev 1981; Gauthier and Bonnet 1990). In general, these equations include Richtmyer's terms, where $a / \lambda$ is replaced by an (adjustable) constant, and a drag term that comprises a scale length. The principal differences between such models is the implementation of the scale length. Two phase models are also used to describe the nonlinear interpenetration of the materials.

The turbulent phase has been modeled with variants of a $k \varepsilon$ model. Simple models include equations for the turbulent energy and its dissipation rate, with some closure model (usually in the form of diffusion terms). More sophisticated models retain additional moments of the fluctuating components.

Most of these models have been developed to represent RT instability growth and (to the extent that a shock can be thought of as a very large acceleration) are applied to the RM instability as well. This extension does not yield acceptable results if the interface is RT stable. To overcome this problem, Crowley (1988) adds to the pressure gradient an "artificial viscosity" term that is significant only at a shock front and provides a source for the turbulent energy in both RT unstable and stable cases. Such a model can be made to represent shock-tube data quite well, as shown in Fig. 6.

The validity of any model depends on its ability to represent a variety of experiments with the same parameters. Most of the proposed models can be made to match a class of problems such as (1) an interface subjected to a single shock or to multiple shocks, (2) a particular configuration (RT stable or unstable), (3) laser-driven shocks, or (4) shock tubes, but they are not universal. The principal difficulty is that the models describe one or the other phase of the evolution of the interface, whereas all phases are involved in an experiment. Newer models attempt to describe the whole process by retaining both of the equations that describe the nonlinear and the turbulent phases and establishing a consistent link between them. Such models appear to be more reliable, but they are, as yet, too new to have been thoroughly tested. Spectral transport models have also been proposed (Buckingham 1983; Besnard 1991).

In addition to models that can be reduced to $1-D$ predictions of the state variables in an RM-induced mixed layer, models designed to account for subscale mixing in 2- and 3 $\mathrm{D}$ computations are being sought. Here again, most of the work addresses RT instability. 


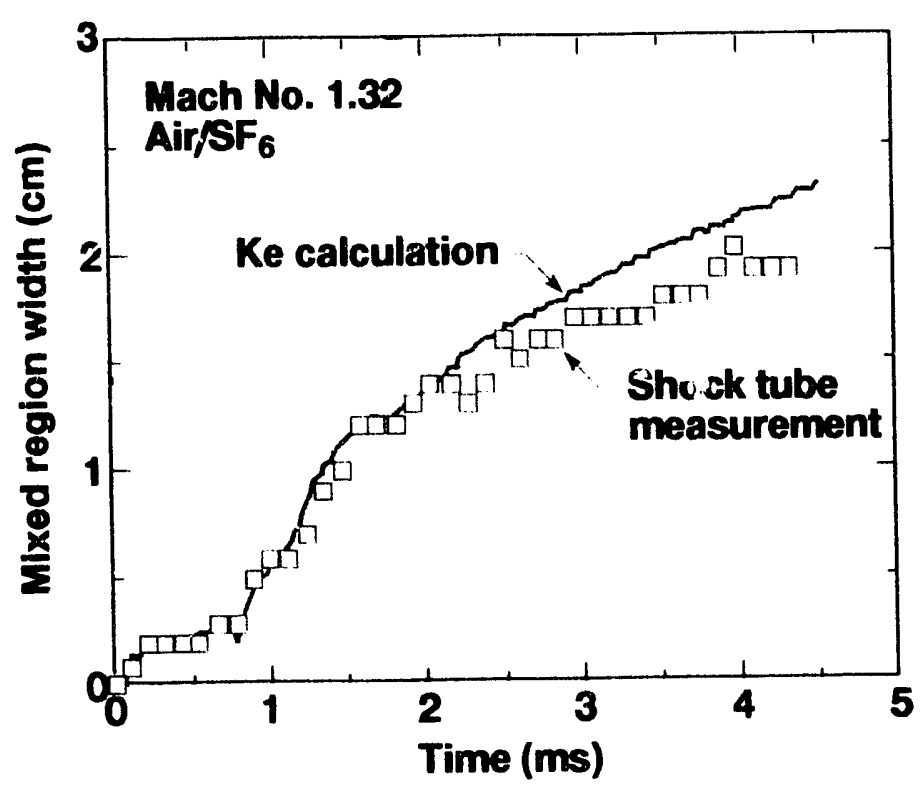

Figure 6. Comparison of experimental data and modeled mix width for a Mach 1.32 shock incident from air into an air/SF 6 interface. $A$ KE model was used with an additional source term to take the shockinduced mixing into account. Model parameters can be selected to match the data quite closely, but they are not universal.

\section{Summary}

In summary, much has been accomplished in the past decade, but there is still room for exciting research in all aspects of the RM mixing problem. It is important to accumulate a data base of sufficiently different experiments and numerical simulations to help fix model parameters and independently verify their universality. If one is to rely to any extent on numerical simulations, it is also necessary to ascertain their reliability by comparing them with known experimental results before using them to describe situations that cannot be tested in a laboratory.

In the experimental arena, better diagnostics will give more detailed, consistent, and reliable data. Future experiments will need to concentrate on configurations arnenable to numerical simulations with well-defined initial conditions, and the effect of extraneous items will have to be quantified.

In the numerical arena, it will be necessary to incorporate all the aspects of the experiments that are being simulated, and 3-D codes should be used more universally, at least until 2- and 3-D results can be reliably correlated.

Models of the inixed region need to be refined to account for all phases of perturbation growth and their transition.

The challenge rcinains to develop a predictive capability.

\section{Acknowletgment}

Space limitatin precludes an exhaustive list of all the esearchers who have contributed to acivances in the field of RM induced mixing. Further references can be found in the works cited here.

Work performed under the auspices of the U.S. Department of Energy by the Lawrence Livermore National Laboratory under Contract No. W-7405-Eng-48. 


\section{References}

Aleshin AN, Gamalii EG, Zaitsev SG, Lazareva EV, Lebo IG, and Rozanov VB (1988) Nonlinear and transitional stages in the onset of the Richtmyer-Meshkov instability, Sov. Tech. Phys. Lett. 14(6): 466-468, AIP translation

Andronov VA, Bakhrakh SM, Meshkov EE, Mokhov VN, Nikiforov VV, Pevnitskii AV, and Tolshmyakov AI (1976) Turbulent mixing at contact surface accelerated by shock waves, Sov. Phys. JETP 44(2): $424-427$

Benjamin RF (1988) Experimental observation of shock stability and shock-induced turbulence. In: Buckingham A, Leith C, and Dannevik W (eds) Proc. Internat. Workshop Phys. Compressible Turbulent Mixing, Springer Verlag Lectures in Engineering Series, New York, Berlin, Heidelberg, in press

Benjamin RF (1989) New diagnostics for shock tube experiments: imaged light scattering. In: Rupert V (ed) Proc. Internat. Exchange on RM and RT Mixing, unpublished

Benjamin RF (1991) Shock and reshock of an unstable fluid interface. In: Wilke N (ed) Third Internat. Workshop Phys. Compressible Turbulent Mixing, AIP Conf. Proc., New York, to be published

Besnard DC, Haas JF, and Rauenzahn RM (1989) Advances in fluid turbulence. In: Proc. CNLS Annu. Conf., May 1988, Elsevier

Besnar's DC ${ }_{y}$ Harlow FH, Rauenzahn RM, and Zemach C (1989) Spectral Transport Model for Varaible Density Turbulent Flow. In: Rupert V (ed) Proc. Internat. Exchange on RM and RT Mixing, unpublished

Bonazza R and Sturtevant B (1991) X-ray densitometry of shock-excited RM instability at an air/xenon interface. Proc. this symposium

Brouillette M and Sturtevant B (1989) Growth induced by multiple shock waves normally incident on plane gaseous interfaces. In: Advances in Fluid Turbulence, Proc. CNLS Annu. Conf., May 1988, Elsevier, New York; also Richtmyer-Meshkov instability at a continuous interface. In: 17th ISSW, AIP Conf. Proc. 208: 284-289

Buckingham AC and Nowak DA (1983) Numerical studies of shock amplified flow instabilities by a hybrid lagrange Euler pseudo-spectral method,.AIAA paper 840263, AIAA, New York

Budzinski J and Zukoski E, California Institute of Technology, Pasadena, Calif. (USA) private communication.

Cavailler C, Croso H, Haas JF, Grandeboeuf P, and Rodriguez G (1991) Results from the Vaujours Vertical Shock Tube. In: Wilke N (ed) Third Internat. Workshop Phys. Compressible Turbulent Mixing, AIP Conf. Proc., New York, to be published

Cavailler C, Mercier P, and Rodriguez G (1989) A new vertical shock tube for RT instability measurements. In: Rupert V (ed) Proc. Internat. Exchange on RM and RT Mixing, unpublished

Cloutman LD (1990), Lawrence Livermore National Laboratory, Livermore, Calif. (USA), private communcation

Cowperthwaite $N$ (1989) 1D, 2D, and 3D calculations of shock tube mixing experiments. In: Rupert V (ed) Proc. Internat. Exchange on RM and RT Mixing, unpublished

Crowley WP (1983), Lawrence Livermore National Laboratory, Livermore, Calif. (USA), private communcation 
Crowley WP (1988) Application of a two-equation turbulence mix model. In:

Buckingham A, Leith C, and Dannevik W (eds) Proc. Internat. Workshop Phys. Compressible Turbulent Mixing, Springer Verlag Lectures in Engineering Series, New York, Berlin, Heidelberg, in press

Gauthier S and Bonnet M (1990) A KE model for turbulent mixing in shock tube flows induced by RT instability. Phys. Fluids A 2(9): 1685-1694

Glimm J, Grove JW, Chen Y, and Li XL (1991) Chaotic mixing at unstable interfaces. In: Wilke N (ed) Third Internat. Workshop Phys. Compressible Turbulent Mixing, AIP Conf. Proc., New York, to be published

Griswold DL (1991) Test Problems. In: Wilke N (ed) Third Internat. Workshop Phys. Compressible Turbulent Mixing, AIP Conf. Proc., New York, to be published

Haas JF (1983) Interaction of weak shock waves and discrete inhomogeneities, CIT Doctoral Thesis

Henderson LF, Coiella P, and Puckett EG (1991) On the refraction of shock waves at a slow-fast gas interface, J. Fluid Mech. 22(4): 1-24

Houas L, Farhat A, and Brun R (1988) Shock induced Rayleigh-Taylor instability in the presence of a boundary layer. Phys.Fluids 31(4): 807-812

Jacobs JW (1991) PLIF flow visualization of shock accelerated light and heavy gas cylinders. In: Wilke N (ed) Third Internat. Workshop Phys. Compressible Turbulent Mixing, AIP Conf. Proc., New York, to be published

Klein RI and Colella P (1989) The interaction of a supernova remnant with interstellar clouds using high order local adaptive mesh refinement methods. Lawrence Livermore National Laboratory, Livermore, Calif. (USA), UCRL-100762

LeLevier $\mathrm{R}$ et al. (1955) Effect of a density gradient on Taylor instability. Lawrence Livermore National Laboratory, Livermnre, Calif. (USA), UCRL 4459 (1955)

Meshkov EE (1969) Instability of a shock wave accelerated interface between two gases. Izv., Akad. Nauk SSSR, Mekh. Zhidk. i Gaz.:151-158, NASA translation TT F-13,074 (1970) R.F.

Meshkov EE, Nikiforov VV, and Tolshmjakov AI (1989) About the structure of turbulent mixing layer at two-gases' interface, accelerated by shock waves. In: Rupert V (ed) Proc. Internat. Exchange on RM and RT Mixing, unpublished

Mikaelian KO (1985) Richtmyer-Meshkov instabilities in stratified fluids. Phys. Rev. A 31(1): $410-419$

Neuvazhaev VE and Yokolev VG (1981) Mixing of a contact boundary retarded by stationary shock waves (trans. from) Zhurnal Prikladnoi Mekhaniki i Technicheskoi Fiziki 2: 85-89; translated by Plenum, New York, pp 211-215

Puckett EG, Colella P, and Heriderson LF (1989) Computations of the refraction of a plane shock wave at a slow-fast gas interface. In: Proc. 17th ISSW, AIP Conf. Proc. 208, AIP, New York, pp 946-950

Rauenzahn RM (1989) Test problem calculations with caveat. In: Rupert V (ed) Proc. In:ernat. Exchange on RM and RT Mixing, unpublished

Read KI (1984) Experimental investigation of turbulent mixing by RT instability. Physica 12D: 45

Richtmyer RD (1960) Taylor instability in shock acceleration of compressible fluids. Comm. Pure Appl. Math. 13: 297-319

Rupert VC (1989) Reference problems. In: Rupert V (ed) Proc. Internat. Exchange on RM and RT Mixing, unpublished; also, (1991) Test Problems. In: Wilke N (ed) Third Internat. Workshop Phys. Compressible Turbulent Mixing, AIP Conf. Proc., New York, to be published 
Sahoda M (1990) Delaunay tetrahedrization in a 3-dimensional free Lagrangian multimaterial code. In: Proc. Next Free Lagrange Conference, to be published

Smith AV (1989) Turbulent mixing in shock tube experiments with a high initial perturbation. In: Rupert V (ed) Proc. Internat. Exchange on RM and RT Mixing, unpublished

Stearman N and Edwards J (1989) Shock tube experiments. In: Rupert V (ed) Proc. Internat. Exchange on RM and RT Mixing, unpublished

Taylor GI (1950) The instability of liquid surfaces when accelerated in a direction perpendicular to their plane. Proc. Roy. Soc. A210: 192-196

Vasilenko AM et al (1988) Experimental research of gravitational instability and turbulization of flow at noble gas interface. In: Buckingham A, Leith C, and Dannevik W (eds) Proc. Internat. Workshop Phys. Compressible Turbulent Mixing, Springer Verlag Lectures in Engineering Series, New York, Berlin, Heidelberg, in press

Wehner MF (1989) Pure Eulerian hydrodynamics calculations of the two standard test problems. In: Rupert V (ed) Proc. Internat. Exchange on RM and RT Mixing, unpublished

Wehner MF (1991) Test Problems. In: Wilke N (ed) Third Internat. Workshop Phys. Compressible Turbulent Mixing, AIP Conf. Proc., New York, to be published

Yang X and Zabuski NJ (1989) Shock induced dipolar vortex structure and compressible turbulence. In: Rupert V (ed) Proc. Internat. Exchange on RM and RT Mixing, unpublished

Youngs DL (1984) Numerical simulation of turbulent mixing by RT instability. Physica 12D: 32

Youngs DL (1989) Three dimensional numerical simulation of turbulent mixing by Rayleigh-Taylor instability. In: Rupert V (ed) Proc. Internat. Exchange on RM and RT Mixing, unpublished

Zaitsev S, Aleshin A, Lazareva E, Titov S, and Cheboyareva E (1991) Experimental investigation of Rayleigh-Taylor and Richtmyer-Meshkov instabilities. In: Wilke N (ed) Third Internat. Workshop Phys. Compressible Turbulent Mixing, AIP Conf. Pro.., New York, to be published

Zaitsev SG, Lazareva EV, Chernukha VV, and Belyaev VM (1985) Intensification of mixing at the interface between media of different densities upon the passage of shock wave through it. Sov. Phys. Dokl. 30: 567

Zufiria JA (1988) Bubble competition in RT instability. Phys. Fluids 31(3): 440-446 

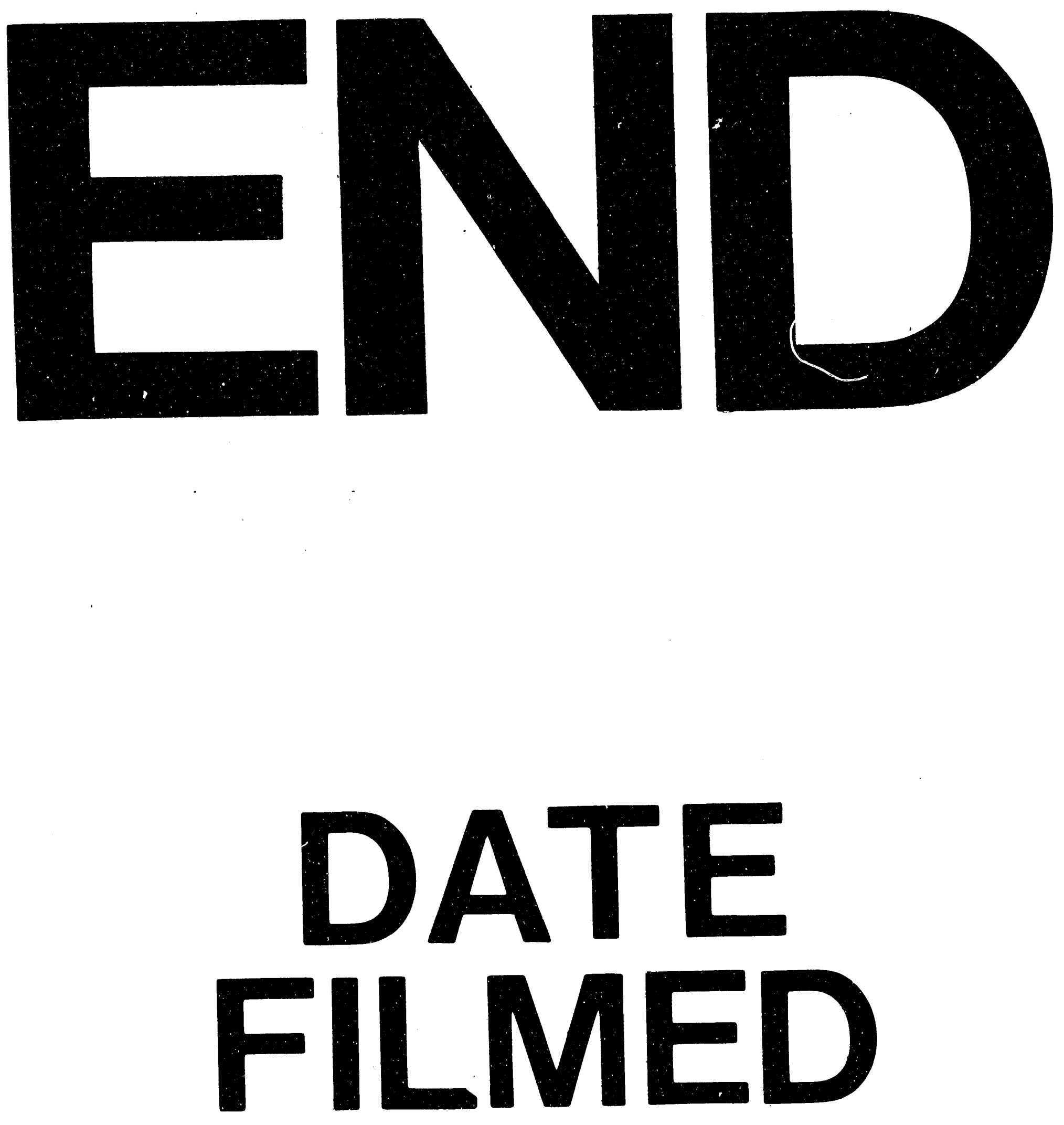

$\neq$

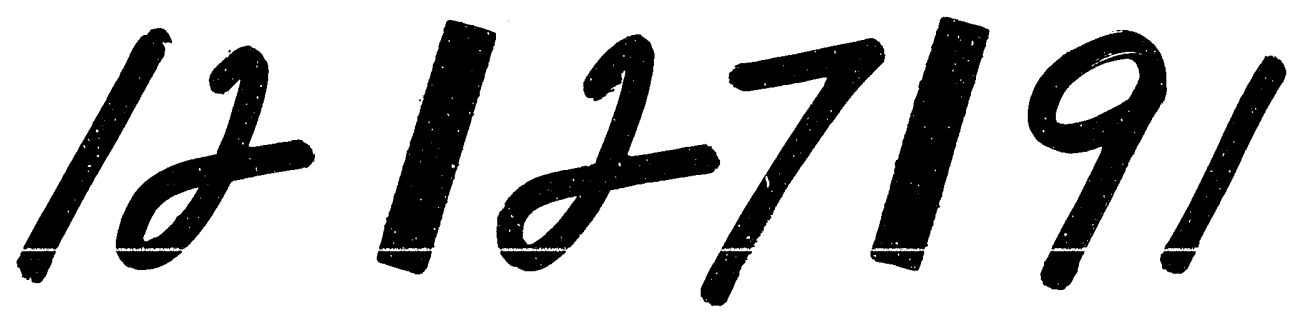


